\title{
Cardiac Troponin T and Hydration Status as Prognostic Markers in Hemodialysis Patients
}

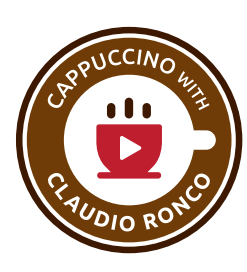

\author{
Krzysztof Hoppe ${ }^{a} \quad K$ rzysztof Schwermer ${ }^{a} \quad$ Patrycja Klysz $^{a} \quad$ Dorota Radziszewska $^{a}$ \\ Peter Sawatiuk $^{a}$ Ewa Baum ${ }^{a}$ Jolanta Kaczmarek ${ }^{a}$ Magdalena Roszak ${ }^{c}$ \\ Malgorzata Kaluzna ${ }^{a}$ Bengt Lindholm ${ }^{b} \quad$ Krzysztof Pawlaczyk $^{a, b} \quad$ Andrzej Oko $^{a}$ \\ ${ }^{a}$ Department of Nephrology, Transplantology, and Internal Medicine, Poznan University of Medical Sciences, Poznan, \\ Poland; 'b Divisions of Renal Medicine and Baxter Novum, Department of Clinical Science, Intervention, and Technology, \\ Karolinska Institutet, Stockholm, Sweden; ' Department of Computer Science and Statistics, Poznan University of \\ Medical Sciences, Poznan, Poland
}

\section{Key Words}

Hemodialysis - End-stage renal disease - Cardiac troponin T. Cardiovascular risk - Traditional risk factors - Nontraditional risk factors · Overhydration · Nutrition

\section{Abstract \\ This study aimed to assess cardiac troponin T (cTnT) and hy- dration state as cardiovascular (CV) risk markers in hemodi- alysis (HD) patients. Two hundred and forty one patients were divided according to HD vintage into two groups: SV (HD $\leq 24$ months) and LV. Water balance was assessed with overhydration (OH\%; bioimpedance analysis) and daily di- uresis (DD); CV dysfunction with CTnT and heart ultrasound; nutrition with subjective global assessment (SGA), choles- terol (TC) and albumin. SV had lower $\mathrm{OH} \%$ (2.8 vs. 3.5, $\mathrm{p}<0.05)$ and higher $\mathrm{DD}(1,161 \mathrm{vs.} 637 \mathrm{ml}, \mathrm{p}<0.001)$, while LV had higher cTnT ( $0.1 \pm 0.04$ vs. $0.1 \pm 0.07 \mathrm{ng} / \mathrm{ml}, \mathrm{p}<0.05)$ and lower interventricular septum thickness (IVS; 13.4 vs. $14.5 \mathrm{~mm}, \mathrm{p}<0.05)$. Nutritional state as reflected by lower TC was worse in LV (184.7 vs. $169.5 \mathrm{mg} / \mathrm{dl}, \mathrm{p}<0.05)$. Mortality was higher in patients in the LV group (15 vs. 27 deaths, $p<$ $0.05)$. $\mathrm{OH} \%$ correlated inversely with albumin $(r=-0.36, p<$}

$0.001), \mathrm{TC}(r=-0.31, \mathrm{p}<0.001)$ and $\mathrm{CTnT}(\mathrm{r}=-0.4, \mathrm{p}<0.001)$. cTnT correlated positively with IVS $(r=0.39, p<0.001)$, SGA $(r=0.23, p=0.001)$ and mortality rate $(r=0.21, p<0.01)$, and negatively with $D D(r=-0.34, p<0.001)$ and albumin $(r=$ $-0.25, p<0.001$ ). Longer dialysis vintage associates with CV dysfunction, overhydration and increased mortality, which may be predicted with $\mathrm{OH} \%$ and $\mathrm{CTnT}$.

Video Journal Club 'Cappuccino with Claudio Ronco' at http://www.karger.com/?doi=376603. @ 2015 s. Karger AG, Basel

\section{Introduction}

Patients with end-stage renal disease (ESRD) on maintenance hemodialysis (HD) are at increased risk of cardiovascular (CV) incidents, which are the most common cause of death among this group being responsible for more than $40 \%$ of mortality; this reaches $50 \%$ in dialysis patients after 3 years of therapy [1]. Cardiovascular risk in the general population depends mainly on well-known both potentially modifiable and non-modifiable factors. The INTERHEART Modifiable Risk Score identifies 9

\section{KARGER 125}

(c) 2015 S. Karger AG, Base

$0253-5068 / 15 / 0402-0139 \$ 39.50 / 0$

E-Mail karger@karger.com

www.karger.com/bpu
Krzysztof Pawlaczyk

Department of Nephrology, Transplantology, and Internal Medicine Poznan University of Medical Sciences, Przybyszewskiego Street 49 PL-60-461 Poznan (Poland)

E-Mail kpawlac@ump.edu.pl 
modifiable factors: lipid abnormalities, smoking, hypertension, diabetes, abdominal obesity, poor psychosocial conditions, lack of physical activity, high alcohol intake and high-risk diet, which together explain almost $95 \%$ of the risk for myocardial infarction incidents in the general population $[2,3]$ although irreversible features such as older age, male gender and genetic predispositions also play a role [2-4]. However, recent analyses of traditional risk factors' influence on cardiovascular events in the population of HD patients do not explain the increased risk of such incidents [4]. The mystery of this fact is hidden in the presence of nontraditional cardiovascular risk determinants, some of them being specific for ESRD patients, which include chronic inflammation, multifactorial anemia, bone-mineral metabolism abnormalities and impaired nutritional state [5-7]. In addition, another negative process not without significance, HD-induced myocardial stunning leading to irreversible heart muscle injuries suggest the existence of another group of CV risk factors, which could be called iatrogenic [8]. The awareness of such significant $\mathrm{CV}$ risk of maintenance HD patients requires searching for useful markers that can predict acute incidents.

Cardiac troponin $\mathrm{T}$ ( $\mathrm{cTn} \mathrm{T}$ ) is unique to the heart and an indicator, superior among other biomarkers, of cardiomyocyte damage [9]. Previous studies ruled out the hypothesis of cTnT elevation as a result of muscular injury in uremic myopathy [10]. As part of the gold standard, the triple model featuring acute chest pain and 12lead electrocardiogram abnormalities has a prominent value in acute myocardial infarction (AMI) diagnosis requiring early coronary intervention [11]. Another concern is that elevated cTnT serum concentrations are detected in most of the patients with ESRD on chronic HD, the natural cause being a decrease in renal clearance of small fragments of this molecule [12]. On the other hand, whereas an increased cTnT concentration is present in a substantial portion of patients with low GFR, a fraction of ESRD subjects without diagnosed CV disease has no detectable levels of serum cTnT. Clinical analyses of patients with chronic heart failure and stable coronary artery disease suggest a prognostic value of cTnT elevation for CV and all-cause mortality $[13,14]$. These observations may suggest an involvement of CV microinjuries affecting CKD and particularly ESRD patients leading to an increase in cTnT as an early manifestation [15]. Previous studies showed an important benefit of cTnT in asymptomatic CV damage detection and therefore acute incidents prediction among the HD population [16-18].
Another essential problem affecting both HD and peritoneal dialysis patients and strongly influencing their quality of life and long-term prognosis is their body composition with a strong emphasis on hydration state and inter-dialytic fluid gain [19]. Even nowadays, the most commonly used methods in conducting routine practice for evaluation of excessive fluid and determining dry body weight include patient's physical examination. However, a novel, thorough and practical technique of body composition measurement both in scientific and routine clinical use has become available. This new solution is the bioimpedance analysis (BIA) [20]. The electric current flows through the fluid components of the body between electrodes attached to patient's head and foot. The voltage to current ratio is the impedance that has two elements, resistance connected with extracellular fluid current flow and reactance, which originates from the indirect flow across cell membranes [20]. The algorithm for proper body composition analysis featuring fluid volume, its location, as well as adipose and lean tissue estimation requires precise patient characteristics including body weight, height, blood pressure and other measurements depending on the BIA device [20].

The aim of this study was to assess the value of cardiac troponin $\mathrm{T}$ and hydration status as markers of $\mathrm{CV}$ risk and long-term survival of patients undergoing maintenance HD therapy and to determine the influence of nontraditional kidney disease-related factors including nutritional state parameters, anemia, hypertension and diabetes mellitus on CV risk and all-cause mortality.

\section{Materials and Methods}

The studied group consisted of 241 patients on maintenance HD ( $81 \mathrm{~F}, 160 \mathrm{M}$, mean age $61.9 \pm 12.5$ years) who were split into two subgroups according to the duration of preceding HD treatment: Group SV (short vintage) had received HD $\leq 24$ months (77 M, $42 \mathrm{~F}$, mean age 62.2 \pm 13.1 years) and Group LV (long vintage) had preceding $\mathrm{HD}>24$ months $(83 \mathrm{M}, 39 \mathrm{~F}, 61.7 \pm 12.0$ years). Characteristics of both groups are shown in table 1 . Patients were followed-up for 30 months. Studied subjects were given full information and each subject provided their written consent. The study was approved by the Ethical Committee at Poznan University of Medical Sciences.

The following measurements focusing on assessments of hydration state, cardiovascular and nutritional state and inflammatory markers, were performed in each individual before the midweek dialysis session. Fluid status parameters featured overhydration to total body weight ratio $(\mathrm{OH} \%)$ assessed using bioimpedance analysis (measured with Fresenius Body Composition Monitor) and residual daily diuresis. The bioimpedance measurement was performed immediately before the HD session, with patients in a 
stable, supine position, using a set of four single-use electrodes placed in doubles on the subject's wrist and ankle on the opposite side to the arterio-venous fistula.

Serum cTnT concentration was measured using Elecsys/cobas e cTnT fourth-generation assay (Roche Diagnostics) and transthoracic echocardiographic examination following available guidelines [21] was performed to assess left ventricle mass parameters: left ventricle wall (LVW) and interventricular septum (IVS) thickness and to evaluate for cardiovascular injury.

For nutritional state assessment, the Subjective Global Assessment (SGA) questionnaire, fat tissue index (FTI), lean tissue index (LTI) - measured using bioimpedance, and total cholesterol (TChol) and albumin (alb) serum concentrations were used. SGA was performed with a dialysis-modified questionnaire following the malnutrition score proposed by Kalantar-Zadeh et al. [22]. It consisted of two parts. First, the patient's medical history featuring weight change in the past 6 months, dietary intake, gastrointestinal symptoms, functional capacity due to nutritional impairment and comorbidities was recorded. Second, a physical examination consisting of anthropometric measurements of signs of fat tissue loss and muscle wasting was performed. A point scale, 1-5, for each category was used; so the total value oscillated between 7 (corresponding with proper nutritional state) and 35 (corresponding with extreme malnutrition). Other laboratory measurements included serum hemoglobin and high-sensitivity C-reactive protein, hsCRP.

Acute cardiovascular incidents during the follow-up comprised acute myocardial infarction and stroke. The mortality rate was not divided into causes. Comorbidities featuring diabetes mellitus (DM) and history of acute CV incidents were taken into account.

Results were calculated using arithmetic mean and standard deviation. Statistical analysis was performed with Statsoft's Statistica 10. Correlations for samples with distinct distribution were evaluated using the Spearman's correlation coefficient, while subgroup comparisons were analyzed using the Mann-Whitney's test. A multivariable analysis of mortality risk factors was also performed.

\section{Results}

Among the 241 included HD patients, 76 patients suffered from DM - 47 from Gr SV, 29 from Gr LV; 40 subjects had a history of myocardial infarction -17 from $\mathrm{Gr}$ SV and 23 from Gr LV; and 19 patients had history of stroke - 6 in Gr SV and 13 in Gr LV (table 1). During the follow-up, 18 episodes of AMI - 7 in Gr SV and 11 in Gr LV - and 6 episodes of stroke - 3 in both groups occurred. Chronic HD therapy lasting longer than 24 months associated with significantly higher rate of deaths (15 vs. 27 , $\mathrm{p}<0.05)$, despite nonsignificant difference in patients' mean age (table 1), as illustrated by Kaplan-Meier's curves (fig. 1).

There were significant differences among the studied groups in hydration state parameters: $\mathrm{OH} \%((\%) 2.8 \pm$

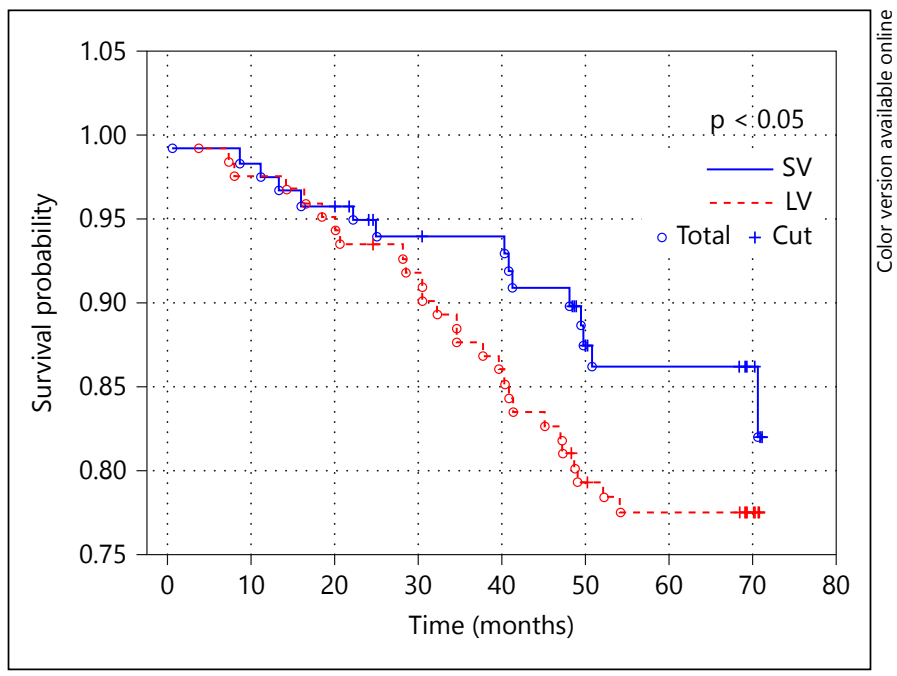

Fig. 1. Kaplan-Meier survival curves for short $(S V ; n=119)$ and long $(\mathrm{LV} ; \mathrm{n}=122)$ dialysis vintage groups.

Table 1. Characteristics of short (SV) and long (LV) dialysis vintage subgroups

\begin{tabular}{llcl}
\hline Characteristics & SV $(\mathrm{n}=119)$ & $\mathrm{LV}(\mathrm{n}=122)$ & $\mathrm{p}$ value \\
\hline Males/females & $77 / 42$ & $83 / 39$ & $\mathrm{NS}(0.66)$ \\
Age, years & $62 \pm 13.1$ & $61.7 \pm 12.0$ & $\mathrm{NS}(0.65)$ \\
HD time span, weeks & $9.3 \pm 6.1$ & $76.2 \pm 40.6$ & $<0.001$ \\
DM & 47 & 29 & $<0.01$ \\
History of AMI & 17 & 23 & NS $(0.59)$ \\
History of stroke & 6 & 13 & NS $(0.47)$ \\
\hline
\end{tabular}

$\mathrm{DM}=$ Diabetes mellitus; $\mathrm{AMI}=$ acute myocardial infarction; NS = non significant.
2.06 vs. $3.5 \pm 2.36, \mathrm{p}<0.02)$ and $\mathrm{DD}((\mathrm{ml}) 1,161.1 \pm 650.7$ vs. $636.7 \pm 584.8, \mathrm{p}<0.000001)$ in SV and LV groups, respectively (table 2 ). Subjects from both groups had persistently elevated cTnT (mean $0.079 \pm 0.056 \mathrm{ng} / \mathrm{ml}$ at time 0 and $0.082 \pm 0.55 \mathrm{ng} / \mathrm{ml}$ after 6 months) considerably exceeding the levels for AMI diagnosis in the general population. Patients in LV group demonstrated significantly higher cTnT $(\mathrm{ng} / \mathrm{ml})$ than SV group measured both at time $0(0.059 \pm 0.037$ vs. $0.097 \pm 0.070, \mathrm{p}<0.03)$ and after 6 months $(0.057 \pm 0.032$ vs. $0.102 \pm 0.069, \mathrm{p}<$ 0.03 ). Heart ultrasound in two groups showed more common incidence of interventricular septum hypertrophy $((\mathrm{mm}) 13.4 \pm 2.2$ vs. $14.5 \pm 2.3, \mathrm{p}<0.02)$ among group LV. No significant differences in other considered parameters $-\mathrm{LVW}((\mathrm{mm}) 13.3 \pm 1.6$ vs. $13.8 \pm 2.0, \mathrm{p}=0.61)$, and $\mathrm{EF}((\%) 54.5 \pm 11.2$ vs. $56.5 \pm 8.2, \mathrm{p}=0.68)$ were 
Table 2. Comparison of short (SV) and long (LV) dialysis vintage subgroups

\begin{tabular}{|c|c|c|c|}
\hline Characteristics & $\mathrm{SV}(\mathrm{n}=119)$ & $\mathrm{LV}(\mathrm{n}=122)$ & $\mathrm{p}$ value \\
\hline AMI, n & 7 & 11 & NS (0.70) \\
\hline Stroke, $\mathrm{n}$ & 3 & 3 & NS (0.98) \\
\hline Deaths, $\mathrm{n}$ & 15 & 27 & 0.045 \\
\hline $\mathrm{OH} \%, \%$ & $2.8 \pm 2.1$ & $3.5 \pm 2.4$ & 0.013 \\
\hline $\mathrm{DD}, \mathrm{ml}$ & $1,161.1 \pm 650.7$ & $636.7 \pm 584.8$ & $<0.000001$ \\
\hline cTnT0, ng/ml & $0.059 \pm 0.037$ & $0.097 \pm 0.070$ & 0.022 \\
\hline cTnT6, ng/ml & $0.057 \pm 0.032$ & $0.102 \pm 0.069$ & 0.026 \\
\hline $\mathrm{LVW}, \mathrm{mm}$ & $13.3 \pm 1.6$ & $13.8 \pm 2.0$ & NS $(0.61)$ \\
\hline IVS, $\mathrm{mm}$ & $13.4 \pm 2.2$ & $14.5 \pm 2.3$ & 0.017 \\
\hline $\mathrm{SBP}, \mathrm{mm} \mathrm{Hg}$ & $137.0 \pm 17.1$ & $138 \pm 17.4$ & NS $(0.40)$ \\
\hline $\mathrm{DBP}, \mathrm{mm} \mathrm{Hg}$ & $82.8 \pm 9.6$ & $83.7 \pm 10.4$ & NS $(0.40)$ \\
\hline SGA score & $11.6 \pm 3.4$ & $10.1 \pm 2.2$ & 0.016 \\
\hline TChol, mg/dl & $184.7 \pm 38.84$ & $169.5 \pm 32.4$ & 0.023 \\
\hline Alb, g/dl & $4.0 \pm 0.4$ & $4.0 \pm 0.3$ & NS (0.85) \\
\hline $\mathrm{BMI}, \mathrm{kg} / \mathrm{m}^{2}$ & $26.7 \pm 3.85$ & $26.1 \pm 3.9$ & NS (0.20) \\
\hline LTI, $\mathrm{kg} / \mathrm{m}^{2}$ & $12.2 \pm 2.3$ & $11.7 \pm 2.1$ & NS (0.18) \\
\hline FTI, $\mathrm{kg} / \mathrm{m}^{2}$ & $13.6 \pm 4.3$ & $13.4 \pm 4.3$ & NS (0.91) \\
\hline $\mathrm{Hb}, \mathrm{g} / \mathrm{dl}$ & $11 \pm 1.2$ & $11 \pm 1.2$ & NS (0.79) \\
\hline
\end{tabular}

$\mathrm{AMI}=$ Acute myocardial infarction; $\mathrm{OH} \%=$ overhydration according to bioimpedance; $\mathrm{DD}=$ daily diuresis; cTnT0 and cTnT6 = cardiac troponin T at baseline and at 6 months, respectively; LVW = left ventricle wall thickness; IVS = interventricular septum thickness; SBP = systolic blood pressure; DBP = diastolic blood pressure; SGA = subjective global assessment score; TChol = total plasma cholesterol; Alb = serum albumin; BMI = body mass index; LTI = lean tissue index according to bioimpedance; FTI = fat tissue index according to bioimpedance; $\mathrm{Hb}=$ hemoglobin; $\mathrm{NS}=$ non significant.

Table 3. Correlations of ECHO parameters with cardiac troponin $\mathrm{T}$, estimated degree of overhydration and all-cause mortality in the combined material of both SV and LV patients ( $\mathrm{n}=241 \mathrm{HD}$ patients)

\begin{tabular}{llll}
\hline & EF & IVS & LVW \\
\hline cTnT0 & $\mathrm{r}=-0.057, \mathrm{NS}(\mathrm{p}=0.55)$ & $\mathrm{r}=0.39, \mathrm{p}=0.000076$ & $\mathrm{r}=0.48, \mathrm{p}=0.000009$ \\
OH\% & $\mathrm{r}=-0.04, \mathrm{NS}(\mathrm{p}=0.62)$ & $\mathrm{r}=0.26, \mathrm{p}=0.0043$ & $\mathrm{r}=0.26, \mathrm{p}=0.019$ \\
All-cause mortality & $\mathrm{r}=-0.23, \mathrm{p}=0.0072$ & $\mathrm{r}=0.09, \mathrm{NS}(\mathrm{p}=0.33)$ & $\mathrm{r}=0.06, \mathrm{NS}(\mathrm{p}=0.59)$ \\
\hline
\end{tabular}

$\mathrm{EF}=$ Ejection fraction; IVS = interventricular septum thickness; LVW = left ventricle wall thickness; cTnT0 = cardiac troponin $\mathrm{T}$ at baseline; $\mathrm{OH} \%$ = overhydration according to bioimpedance; NS = non significant.

observed. Patients from Gr LV had lower TChol ((mg/dl) $184.7 \pm 38.3$ vs. $169.5 \pm 32.4, \mathrm{p}<0.03)$, whereas albumin was similar in the two groups $((\mathrm{g} / \mathrm{dl}) 4.0 \pm 0.4$ vs. $4.0 \pm 0.3$, $\mathrm{p}=0.85)$. However, the SGA questionnaire revealed slightly better nutritional condition of Gr LV patients (SGA score $11.6 \pm 3.4$ vs. $10.1 \pm 2.2, \mathrm{p}<0.02$ ). Comparison of subgroups is shown in table 2 .

The degree of overhydration associated with all measured nutritional state indicators except the lean tissue index. In the combined material of both SV and LV patients, statistically significant negative correlations be- tween $\mathrm{OH} \%$ and nutritional state parameters were found for BMI $(\mathrm{r}=-0.33, \mathrm{p}<0.000001)$, fat tissue index $(\mathrm{r}=$ $-0.38, \mathrm{p}<0.000001)$, serum albumin $(\mathrm{r}=-0.36$, $\mathrm{p}<$ $0.000001)$ and TChol ( $\mathrm{r}=-0.31, \mathrm{p}<0.000006)$, and a positive correlation was found for SGA $(r=0.13, \mathrm{p}<0.05)$. Another essential group of correlations concerned $\mathrm{OH} \%$ and myocardial injury markers (table 3). A raise in $\mathrm{OH} \%$ was associated with $\mathrm{CV}$ system parameters: cTnT ( $\mathrm{r}=$ $0.39, \mathrm{p}<0.000001)$, IVS $(\mathrm{r}=0.26, \mathrm{p}<0.005)$ and LVW $(\mathrm{r}=0.26, \mathrm{p}<0.02)$. The $\mathrm{c} \operatorname{TnT}$ correlated positively with echocardiographic indicators of myocardial injury - 
Table 4. Logistic regression analysis of all-cause mortality markers

\begin{tabular}{ccll}
\hline Variable & OR & $95 \% \mathrm{CI}$ & p value \\
\hline Model 1 & & & 0.001 \\
cTnT & 41.25 & $1.165-1,460.574$ & 0.041 \\
OH\% & 1.180 & $1.030-1.352$ & 0.017 \\
\hline Model 2 & & & 0.002 \\
cTnT & 66.692 & $1.477-3,011.44$ & 0.031 \\
Alb & 0.416 & $0.174-0.993$ & 0.048 \\
\hline Model 3 & & & 0.007 \\
cTnT & 1.540 & $0.013-179.235$ & 0.857 \\
OH\% & 1.123 & $0.920-1.372$ & 0.247 \\
Alb & 0.280 & $0.086-0.912$ & 0.035 \\
TChol & 0.998 & $0.981-1.015$ & 0.830 \\
IVS & 1.157 & $0.948-1.412$ & 0.148 \\
\hline
\end{tabular}

$\mathrm{OR}=$ Odds ratio $\mathrm{CI}=$ confidence interval; $\mathrm{cTnT}=$ cardiac troponin $\mathrm{T} ; \mathrm{OH} \%$ = overhydration according to bioimpedance; $\mathrm{Alb}=$ serum albumin; TChol = total plasma cholesterol; IVS = interventricular septum thickness.

$\operatorname{LVW}(\mathrm{r}=0.48, \mathrm{p}<0.00001)$ and IVS $(\mathrm{r}=0.38, \mathrm{p}<0.00008)$. We also observed inverse correlations between $c T n T$ concentration and nutritional indices: SGA $(r=-0.23$, $\mathrm{p}<0.002)$, alb $(\mathrm{r}=-0.25, \mathrm{p}<0.0006)$, TChol $(\mathrm{r}=-0.23$, $\mathrm{p}=0.002)$, and LTI $(\mathrm{r}=-0.14, \mathrm{p}<0.05)$. There was no correlation between $\mathrm{CTnT}$ and traditional CV risk markers: BMI, gender or age.

The all-cause mortality rate among HD patients was increased along with elevation of $\mathrm{OH} \%(\mathrm{r}=0.16, \mathrm{p}=0.01)$, $\mathrm{cTnT}(\mathrm{r}=0.20, \mathrm{p}<0.004)$, hsCRP $(\mathrm{r}=0.22, \mathrm{p}<0.005)$ and decrease of hemoglobin $(r=-0.19, p<0.03)$; however, it was independent of age, systolic and diastolic blood pressure or albumin concentration. EF appeared to be the only echocardiographic parameter directly related to all-cause mortality $(\mathrm{r}=-0.23, \mathrm{p}=0.072)$. Only a positive tendency was observed between AMI incidents and two potential $\mathrm{CV}$ risk prediction markers $-\mathrm{hsCRP}(\mathrm{r}=0.15, \mathrm{p}=0.05)$ and $\mathrm{c} \operatorname{TnT}(\mathrm{r}=0.15, \mathrm{p}=0.075)$. However, there was no relation between AMI and LV dimensions - IVS and LVW. We observed no significant correlations considering other echocardiographic parameters (table 3).

Multivariable logistic regression analysis (table 4) was performed to identify markers affecting all-cause mortality. Various logistic regression models featuring cTnT, $\mathrm{OH} \%$, alb, TChol, IVS, EF and presence of DM were created. Most of them appeared to be statistically nonsignificant for the whole models or for each variable. The separate models for Gr SV and Gr LV were nonsignificant due to the low mortality rate of Gr SV. Model $1(\mathrm{p}=0.0011)$ showed that increase of cTnT (OR 41.25, 95\% CI 1.165$1,460.574 ; \mathrm{p}=0.041)$ and $\mathrm{OH} \%(\mathrm{OR} 1.18,95 \%$ CI 1.030 $1.352 ; \mathrm{p}=0.017)$ was connected with higher risk of death within 2 years. Model $2(\mathrm{p}=0.002)$ featuring cTnT (OR $66.692,95 \%$ CI 1.477-3,011.44; $\mathrm{p}=0.031$ ) and alb (OR $0.416,95 \%$ CI $0.174-0.993 ; \mathrm{p}=0.048)$ revealed their value as mortality predictors in patients on HD.

\section{Discussion}

Our results revealed a negative association of long HD vintage with both patients' hydration state and cardiovascular system condition and the inseparable relation between these complications. Markers of myocardial injury, cardiac troponin $\mathrm{T}$ and echocardiographic parameters of left ventricle mass were increased in both groups; however, longer preceding HD time span was associated with signs of more significant adverse processes. Although this difference may be due to the fact that DM was more common in the SV group, a long history of HD therapy inevitably leads to the reduction of residual daily diuresis, which is followed by increasing inter-dialytic fluid gains. Interestingly, the influence of longer HD-time span on CV system parameters appeared to manifest itself in heart muscle remodeling illustrated with increase of IVS rather than in decline of EF. There are two main reasons for these processes to occur. First is the cardiotoxicity of arterio-venous fistula. Another - the crucial point of this study - is persistent overhydration which according to our data had an impact on compensatory cardiovascular strain, resulting in an increase of LV mass parameters and myocardial microinjuries reflected in cTnT elevation. However, when the compensatory mechanisms fail, chronic heart failure develops and leads to excessive fluid redistribution with blood pressure decrease and peripheral and central edema.

The results do not clearly confirm any deteriorative influence of long HD vintage on the nutritional condition. From the clinical point of view, we may only suggest that the decreased TC level among LV group patients is a result of malnutrition rather than of proper lipid control. However, SGA and albumin results do not confirm this hypothesis.

Our study revealed higher mortality in the LV group despite the SV group having no significant advantage of myocardial infarctions or strokes and higher frequency of DM. This observation strongly confirms the negative influence of HD vintage on survival. Moreover, the advantage in Gr SV for patients with DM, which leads to other 
comorbidities, may to some degree explain the cause of a higher SGA score in this group. Comparable mean age and both SBP, and DBP, which belong to the group of traditional risk factors, strongly confirm the hypothesis that nontraditional kidney disease-derived and HD-induced factors play crucial roles in the high mortality rate in these patients. Presented data showed that increased levels of hsCRP, overhydration, low hemoglobin and, in particular, an elevated serum cTnT concentration, are indicators of poor prognosis and associate with higher mortality in HD patients. cTnT was elevated above the level of AMI diagnosis for the general population in almost $95 \%$ of the studied subjects from both groups. Although persistent elevation of cTnT is common in ESRD patients, particularly in those with DM who frequently have atypical chest pain due to peripheral neuropathy and CV abnormalities, the measurement of cTnT appears to be a useful prognostic tool.

The current finding of the negative influence of overhydration requiring higher $\mathrm{HD}$ ultrafiltration rate on $\mathrm{CV}$ system follows the observations of Burton et al. [8], suggesting the existence of iatrogenic HD-induced $\mathrm{CV}$ risk factors. Velasco et al. [23] performed an experiment to confirm the negative influence of HD on patients' fluid balance. Their study of thrice-weekly hemofiltration, a more physiological dialytic method avoiding substantial fluid imbalance, instead of HD, resulted in improved cTnT and N-terminal pro-brain natriuretic peptide values. In the current study, a lack of correlation between SBP, DBP and cTnT elevation on all-cause mortality indirectly confirms the results of Velasco et al. [23], suggesting that large interdialytic weight gains requiring increase of UF lead to greater intra- and post-dialytic BP drops, which most likely are as unfavorable as increased but stable BP. Our finding of no direct correlation between cTnT, $\mathrm{OH} \%$ and the incidence of AMI (only a tendency for cTnT) is in contrast to previous studies $[16,24]$. A previous study by Pianta et al. [25] revealed no relationship between cTnT and left ventricle mass parameters. We found that both IVS and LVW thickness increase along with a higher cTnT level. We also documented a higher specificity of cTnT and $\mathrm{OH} \%$ in comparison with the echocardiographic LV dimensions. Another essential value of cTnT monitoring confirmed by previous reports $[16,18,26-28]$ is a substantial association with the long-term all-cause mortality. Khan et al. [28] showed in a meta-analysis the prognostic value of both cardiac troponins T and I among ESRD patients; 15 out of $16 \mathrm{cTnT}$ studies found an independent association of elevation of this marker on all-cause and cardiovascular mortality. Moreover, the association in- creased with dialysis duration and follow-up length. Several studies also reported the association of cTnT with markers of left ventricle hypertrophy, whereas the history of $\mathrm{CV}$ disease or diabetes mellitus had no impact on cTnT and all-cause mortality [28]. All these observations are in concordance with our results suggesting the usefulness and advantage of cTnT in mortality prediction.

Another complication of long-term maintenance HD relates to nutritional status. The analysis in our study suggests a noteworthy detrimental association of dialysis vintage with patients' nutritional condition. We postulate that a lower mean cholesterol concentration in the LV group is a result of malnutrition rather than of proper lipid balance. Persistent overhydration was significantly and adversely associated with the nutritional state. It was reflected in several subjective and objective indicators using clinical and laboratory measurements featuring BMI, FTI, SGA, albumin and total cholesterol levels. The negative relationship between $\mathrm{BMI}$ and $\mathrm{OH} \%$ seems noteworthy. Despite similar age, gender, systolic and diastolic blood pressures, no significant difference was noticed in acute cardiovascular incidents and DM predominance among the group SV subjects; however, all-cause mortality was higher in group LV. We revealed the impact of overhydration in HD patients on poor nutritional state illustrated with several parameters including either laboratory indicators - albumin and total cholesterol - or body composition indexes - BMI, FTI, LTI. Demirci et al. proposed similar procedures for subjects undergoing peritoneal dialysis. Their study revealed a negative relation between fluid overload and serum albumin [29].

To conclude, cardiac troponin $\mathrm{T}$ seems to be a valuable marker for $\mathrm{CV}$ risk evaluation and all-cause mortality prediction suggesting that the routine use of sequential cTnT measurements in HD patients, either for the abovementioned reasons or to establish baseline values for each subject for proper acute diagnosis of coronary incidents, could be of value. This study also confirmed results of previous studies of the usefulness of the overhydration index $\mathrm{OH} \%$ and hsCRP as predictors of all-cause mortality risk in ESRD patients. Our observations also confirmed that parameters reflecting poor nutritional status, and not high cholesterol or high BMI, associate with increased cardiac injury parameters and death rate in HD patients; this is in line with the concept of reverse epidemiology of CV risk factors in ESRD patients [30, 31]. Thus, our results indicate the importance of nontraditional risk factors, such as persistent overhydration, malnutrition, inflammation and anemia, over traditional risk factors as predictors of $\mathrm{CV}$ injury in $\mathrm{HD}$ patients. 


\section{Acknowledgments}

We thank all those among the clinical and laboratory staff who contributed to the study. Baxter $\mathrm{N}$ ovum is the result of a grant from Baxter Healthcare Corporation to Karolinska Institutet.

\section{Disclosures}

Bengt Lindholm is employed by Baxter Healthcare Corporation. None of the other authors declare any conflict of interest.

\section{References}

1 Collins AJ, Foley RN, et al: United States renal data system 2011 annual data report: atlas of chronic kidney disease and end-stage renal disease in the United States. Am J Kidney Dis 2012;59(suppl 1):A7.e1-e420.

2 McGorrian C, Yusuf S, et al: Estimating modifiable coronary heart disease risk in multiple regions of the world: the INTERHEART modifiable risk score. Eur Heart J 2011;32 581-589.

3 Anand SS, Islam S, et al: Risk factors for myocardial infarction in women and men: insights from the INTERHEART study. Eur Heart J 2008;29:932-940.

4 Parfrey PS, Foley RN: The clinical epidemiology of cardiac disease in chronic renal failure. J Am Soc Nephrol 1999;10:1606-1615.

5 Wanner C, Metzger T: C-reactive protein a marker for all-cause and cardiovascular mortality in haemodialysis patients. Nephrol Dial Transplant 2002;17(suppl 8):29-32; discussion 39-40.

6 Massy ZA, Barreto DV, et al: Uraemic toxins for consideration by the cardiologist-Beyond traditional and non-traditional cardiovascular risk factors. Atherosclerosis 2010;211: 381-383.

7 Levin A, Djurdjev O, et al: Cardiovascular disease in patients with chronic kidney disease: getting to the heart of the matter. Am J Kidney Dis 2001;38:1398-1407.

8 Burton JO, Jefferies HJ, et al: Hemodialysisinduced cardiac injury: determinants and associated outcomes. Clin J Am Soc Nephrol 2009;4:914-920.

9 Twerenbold R, Jaffe A, et al: High-sensitive troponin $\mathrm{T}$ measurements: what do we gain and what are the challenges? Eur Heart J 2012; 33:579-586.

10 Ricchiuti V, Voss EM, et al: Cardiac troponin $\mathrm{T}$ isoforms expressed in renal diseased skeletal muscle will not cause false-positive results by the second generation cardiac troponin $\mathrm{T}$ assay by Boehringer Mannheim. Clin Chem 1998;44:1919-1924.
11 Thygesen K, Alpert JS, et al: Third universal definition of myocardial infarction. Eur Heart J 2012;33:2551-2567.

12 Diris JH, Hackeng CM, et al: Impaired renal clearance explains elevated troponin $\mathrm{T}$ fragments in hemodialysis patients. Circulation 2004; 109:23-25.

13 Latini R, Masson S, et al: Prognostic value of very low plasma concentrations of troponin $\mathrm{T}$ in patients with stable chronic heart failure. Circulation 2007;116:1242-1249.

14 Omland T, de Lemos JA, et al: A sensitive cardiac troponin $\mathrm{T}$ assay in stable coronary artery disease. N Engl J Med 2009;361:25382547.

15 Dubin RF, Li Y, et al: Predictors of high sensitivity cardiac troponin $\mathrm{T}$ in chronic kidney disease patients: a cross-sectional study in the chronic renal insufficiency cohort (CRIC). BMC Nephrol 2013;14:229.

16 Hassan HC, Howlin K, et al: High-sensitivity troponin as a predictor of cardiac events and mortality in the stable dialysis population. Clin Chem 2014;60:389-398.

17 Wang AY, Lam CW, et al: Prognostic value of cardiac troponin $\mathrm{T}$ is independent of inflammation, residual renal function, and cardiac hypertrophy and dysfunction in peritoneal dialysis patients. Clin Chem 2007;53:882889.

18 Hickman PE, McGill DA, et al: Prognostic efficacy of cardiac biomarkers for mortality in dialysis patients. Intern Med J 2009;39:812-818.

19 Devolder I, Verleysen A, et al: Body composition, hydration, and related parameters in hemodialysis versus peritoneal dialysis patients. Perit Dial Int 2010;30:208-214.

20 Tattersall J: Bioimpedance analysis in dialysis: state of the art and what we can expect. Blood Purif 2009;27:70-74.

21 Lang RM, Bierig M, et al: Recommendations for chamber quantification. Eur J Echocardiogr 2006;7:79-108.

22 Kalantar-Zadeh K, Kleiner M, et al: A modified quantitative subjective global assessment of nutrition for dialysis patients. Nephrol Dial Transplant 1999;14:1732-1738.

23 Velasco N, Chamney P, et al: Optimal fluid control can normalize cardiovascular risk markers and limit left ventricular hypertrophy in thrice weekly dialysis patients. Hemodial Int 2012;16:465-472.

24 Roberts MA, Hare DL, et al: Serial increased cardiac troponin $\mathrm{T}$ predicts mortality in asymptomatic patients treated with chronic haemodialysis. Ann Clin Biochem 2009;46: 291-295.

25 Pianta TJ, Horvath AR, et al: Cardiac highsensitivity troponin T measurement: a layer of complexity in managing haemodialysis patients. Nephrology (Carlton) 2012;17:636641.

26 Dierkes J, Domröse U, et al: Cardiac troponin $\mathrm{T}$ predicts mortality in patients with endstage renal disease. Circulation 2000;102: 1964-1969.

27 Ooi DS, Zimmerman D, et al: Cardiac troponin $\mathrm{T}$ predicts long-term outcomes in hemodialysis patients. Clin Chem 2001;47:412417.

28 Khan NA, Hemmelgarn BR, et al: Prognostic value of troponin $\mathrm{T}$ and I among asymptomatic patients with end-stage renal disease: a meta-analysis. Circulation 2005;112:30883096.

29 Demirci MS, Demirci C, et al: Relations between malnutrition-inflammation-atherosclerosis and volume status. The usefulness of bioimpedance analysis in peritoneal dialysis patients. Nephrol Dial Transplant 2011;26: 1708-1716.

30 Kalantar-Zadeh K, Block G, et al: Reverse epidemiology of cardiovascular risk factors in maintenance dialysis patients. Kidney Int 2003;63:793-808.

31 Kalantar-Zadeh K, Block G, et al: Reverse epidemiology of conventional cardiovascular risk factors in patients with chronic heart failure. J Am Coll Cardiol 2004;43:1439_ 1444 